\title{
Improved DC Offset Error Compensation Algorithm in Phase Locked Loop System
}

\author{
Chang-Seok Park* and Tae-Uk Jung ${ }^{\dagger}$
}

\begin{abstract}
This paper proposes a dc error compensation algorithm using dq-synchronous coordinate transform digital phase-locked-loop in single-phase grid-connected converters. The dc errors are caused by analog to digital conversion and grid voltage during measurement. If the dc offset error is included in the phase-locked-loop system, it can cause distortion in the grid angle estimation with phase-locked-loop. Accordingly, recent study has dealt with the integral technique using the synchronous reference frame phase-locked-loop method. However, dynamic response is slow because it requires to monitor one period of grid voltage. In this paper, the dc offset error compensation algorithm of the improved response characteristic is proposed by using the synchronous reference frame phase-locked-loop. The simulation and the experimental results are presented to demonstrate the effectiveness of the proposed dc offset error compensation algorithm.
\end{abstract}

Key words: DC offset error, Compensator, PLL, Grid-connected converter, SRF

\section{Introduction}

These days, fossil energy sources such as coal and gas become increasingly deficient, and greenhouse gas emissions cause a large number of environmental pollution problems. Therefore, research about on renewable energy sources has been focused recently [1-4].

The Phase-Lock-Loop (PLL) of the Single-phase Gridconnected Converter system (SGC) is a key issue for power quality instruments $[5,6]$. It is also widely used for control of the electrical machines [7], the grid side power electronic converters of the renewable and distributed generation systems [8]. For the higher power quality of the SGC system, the SGC requires the grid information such as the grid voltage level, amplitude, phase, and frequency [9].

The information of grid is measured by the voltage sensor and then, the measured voltage is input as the signal of digital controller through Low-Pass-Filters (LPFs) and the Analog and Digital Conversion (ADC). In this process, the voltage measurement errors can be occurred due to non-linearity of the voltage sensor, the thermal variation of the analog electric device, and the quantization error of the $\operatorname{ADC}[10,11]$.

In case of the SGC system including the error of the voltage sensor and dc offset during $\mathrm{ADC}$, the measured grid voltage should be contained dc offset and estimated grid angle of PLL should also be distorted. When it comes to use the synchronous coordinate conversion, dc offset could be removed by adding LPFs on synchronous d-axis voltage $[12,13]$. However, estimated grid frequency and

$\dagger \quad$ Corresponding Author: Dept. of Electrical Engineering, Kyungnam University, Korea. (tujung@kyungnam.ac.kr)

* Dept. of Electrical Engineering, Kyungnam University, Korea. (chang8793@naver.com)

Received: December 17, 2015; Accepted: August 6, 2016 synchronous dq-axis voltages have the same ripples with the grid frequency. It is very difficult to filter them out using an extremely low bandwidth [14].

In [15], the focus is on rejecting the dc offset in the single-phase synchronous reference frame PLL. This algorithm can be implemented by a simple integral operation and the proportional-integral (PI) controller without additional hardware. The input signal of the PLL is integrated over two half-cycles. The obtained results are then subtracted from each other and passed through a PI controller. The output of this PI controller, which is an estimation of the input dc component, is subtracted from the PLL input to cancel its dc component. However, dynamic response is slow because it requires to monitor one period of grid voltage. Therefore, there is a disadvantage of dynamic characteristic because dc offset estimation is determined after one period of grid frequency.

This paper proposes the dc offset compensation algorithm which can estimate and remove the dc offset error without a delay of one period of grid voltage.

The proposed method shows improved dynamic response because the dc offset error is continuously estimated by transforming the synchronous $d$-axis voltage to SRF without monitoring one cycle time of grid voltage. The simulation and the experimental results are presented to demonstrate the effectiveness of the proposed compensation dc offset error compensation algorithm.

\section{System Modeling}

\section{1 d-q SRF PLL in grid voltage measurement}

Fig. 1 shows the system configuration of the SGC 


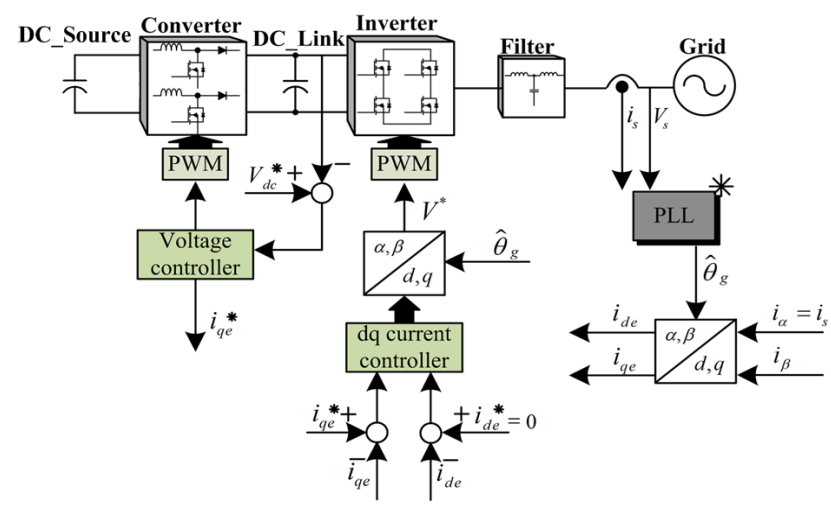

Fig. 1. System structure of SGC

including power circuits and control algorithm.

PLL system is used for detecting the grid angle. The single phase inverter controls the DC voltage and grid current through PI controller. In the single-phase gridconnected inverter, the synchronous $\mathrm{d}-\mathrm{q}$ axis voltage is required for using PLL and the virtual axis is generated by sensing the grid voltage in order to convert the grid voltage into the synchronous d-q axis.

As shown in Fig. 1, the grid components such as the grid voltage level, amplitude, phase and frequency are necessarily required for the PLL in grid connected system. The components of the grid voltage for the PLL are easily obtained from the grid through the voltage sensor.

The ideal grid voltage equation is calculated in (1).

$$
v_{\text {grid }}=-V_{m} \sin \omega_{g} t
$$

where, $V_{m}$ is the grid voltage peak value, $\omega_{g}$ is the grid angular frequency respectively.

An overview of various grid synchronization techniques is available in $[16,17]$. Among them, d-q SRF PLL method is widely used.

In $d-q$ SRF PLL system, single-phase signal is transformed to $\alpha, \beta$ frame by generating virtual voltage which is delayed by $90^{\circ}$ from the grid voltage and can be achieved by using digital all pass filter. The measured grid voltage $v_{\text {grid }}$ is set to be $\alpha$ and $\beta$ is the virtual voltage through the digital all pass filter. And then, $\alpha, \beta$ are transformed to $d-q$ SRF by the Park transform. In this paper, the synchronous $\mathrm{d}$-axis is set a reactive power factor to control to zero and the q-axis is set the effective power factor to control the voltage.

Through $d-q$ SRF PLL, synchronous $d$-axis voltage is controlled to zero so that PLL could estimate the accurate phase and amplitude information of the grid voltage. This is only possible under ideal grid sensing value.

However, the sensed grid voltage $v_{\text {grid }}$ is included some errors which is generated by the voltage sensor because of the non-linearity of the sensors, the thermal variation of the analog electric device and the quantization error of the ADC.

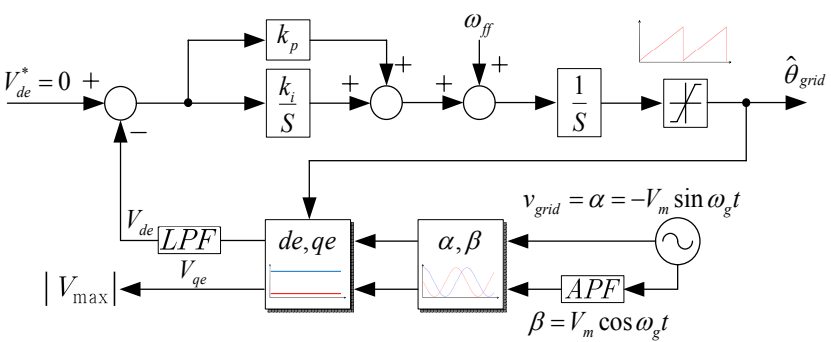

Fig. 2. Basic block diagram of the SRF PLL

\section{Generation of dc offset \& scaling errors}

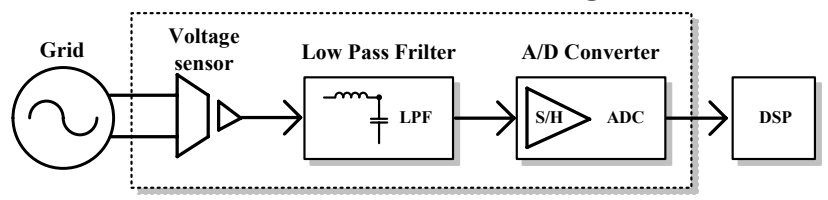

Fig. 3. Path of the grid voltage measurement

Fig. 3 shows the general block diagram of the voltage sensing process about the influence of the grid measurement errors. The measured grid voltage by voltage sensor are various like the dc offset error and the scaling error.

The detection of grid voltage in the SGC system is necessary. However, dc offset is contained in the measured voltage, the synchronous $d-q$ axis voltage should be included the $\mathrm{AC}$ component when the grid voltage and virtual axis are converted to synchronous coordination.

The grid angle estimation can be carried out by controlling the synchronous d-axis voltage constantly but if the d-axis voltage is included AC component by dc offset, the grid angle estimation is distorted by the error.

Considering measurement errors, $v_{\text {grid }}$ can be rewritten by (2).

$$
v_{\text {grid }}=-\Delta_{s} V_{m} \sin \omega_{g} t+\Delta_{\text {off }}
$$

where, $\Delta_{s}$ is the scaling error, $\Delta_{\text {off }}$ is the dc offset error component respectively.

Therefore, $\alpha, \beta$ can be calculated by (3).

$$
\left[\begin{array}{l}
\alpha_{\text {err }} \\
\beta_{\text {err }}
\end{array}\right]=\left[\begin{array}{l}
-\Delta_{s} V_{m} \sin \omega_{g} t+\Delta_{\text {off }} \\
\Delta_{s} V_{m} \cos \omega_{g} t+\Delta_{\text {off }}
\end{array}\right]
$$

In order to obtain the estimated grid angle, frequency and amplitude for the SRF PLL, $\alpha \beta$-axis voltages are transformed to the $d q$-axis synchronous voltage by Park matrix. The transformation matrix is depended on estimation grid angle $\hat{\theta}_{g}$. The transformation Park matrix $T\left(\hat{\theta}_{g}\right)$ can be the following (4).

$$
T\left(\hat{\theta}_{g}\right)=\left[\begin{array}{cc}
\cos \hat{\theta}_{g} & \sin \hat{\theta}_{g} \\
-\sin \hat{\theta}_{g} & \cos \hat{\theta}_{g}
\end{array}\right]
$$


where, $\hat{\theta}_{g}$ is the estimated grid angle.

Considering the errors, the synchronous $d$-axis $V_{d e \text { err }}$ and synchronous $q$-axis voltages $V_{q e_{-} e r r}$ can be obtained as follows (5).

$$
\begin{aligned}
{\left[\begin{array}{l}
V_{d e_{-} e r r} \\
V_{q e_{-} e r r}
\end{array}\right] } & =\left[\begin{array}{cc}
\cos \hat{\theta}_{g} & \sin \hat{\theta}_{g} \\
-\sin \hat{\theta}_{g} & \cos \hat{\theta}_{g}
\end{array}\right]\left[\begin{array}{l}
\alpha_{\text {err }} \\
\beta_{\text {err }}
\end{array}\right] \\
& =\left[\begin{array}{cc}
\cos \hat{\theta}_{g} & \sin \hat{\theta}_{g} \\
-\sin \hat{\theta}_{g} & \cos \hat{\theta}_{g}
\end{array}\right]\left[\begin{array}{l}
-\Delta_{s} V_{m} \sin \omega_{g} t+\Delta_{o f f} \\
\Delta_{s} V_{m} \cos \omega_{g} t+\Delta_{\text {off }}
\end{array}\right]
\end{aligned}
$$

For getting the estimated grid angle, frequency and amplitude, synchronous $d$-axis voltage has to be converged to zero. However, synchronous $d$-axis voltage is not able to be converged to zero due to the dc offset error $\Delta_{\text {off }}$.

From (5), the synchronous $d q$-axis voltages including the scaling error and the dc offset errors can be calculated by (6), (7).

$$
\begin{gathered}
{\left[\begin{array}{c}
V_{\text {de_err }} \\
V_{q e_{-} e r r}
\end{array}\right]=\left[\begin{array}{c}
-\Delta_{s} V_{m} \sin \theta_{g} \cos \hat{\theta}_{g}+\Delta_{\text {off }} \cos \hat{\theta}_{g} \\
\Delta_{s} V_{m} \sin \theta_{g} \sin \hat{\theta}_{g}-\Delta_{\text {off }} \sin \hat{\theta}_{g} \\
+\Delta_{s} V_{m} \cos \theta_{g} \sin \hat{\theta}_{g}+\Delta_{\text {off }} \sin \hat{\theta}_{g} \\
+\Delta_{s} V_{m} \cos \theta_{g} \cos \hat{\theta}_{g}+\Delta_{o f f} \cos \hat{\theta}_{g}
\end{array}\right]} \\
{\left[\begin{array}{c}
V_{d e_{-} e r r} \\
V_{\text {qe_err }}
\end{array}\right]=\left[\begin{array}{c}
-\Delta_{s} V_{m} \sin \left(\theta_{g}-\hat{\theta}_{g}\right)+\Delta_{\text {off }} \cos \hat{\theta}_{g}+\Delta_{\text {off }} \sin \hat{\theta}_{g} \\
\Delta_{s} V_{m} \cos \left(\theta_{g}-\hat{\theta}_{g}\right)-\Delta_{\text {off }} \sin \hat{\theta}_{g}+\Delta_{\text {off }} \cos \hat{\theta}_{g}
\end{array}\right]}
\end{gathered}
$$

where $\theta_{g}\left(=\omega_{g} t\right)$ is real grid angle.

If the difference between real grid angle and the estimated grid angle $\left(\theta_{g}-\hat{\theta}_{g}\right)$ is too small, the synchronous $d q$-axis voltages can be rewritten by (8).

$$
\left[\begin{array}{c}
V_{d e_{-} e r r} \\
V_{q e_{-} e r r}
\end{array}\right]=\left[\begin{array}{c}
\Delta_{o f f} \cos \hat{\theta}_{g}+\Delta_{\text {off }} \sin \hat{\theta}_{g} \\
\Delta_{s} V_{m}-\Delta_{\text {off }} \sin \hat{\theta}_{g}+\Delta_{\text {off }} \cos \hat{\theta}_{g}
\end{array}\right]
$$

Therefore, the error values of the converted synchronous $d q$-axis voltage can be confirmed that it has a component of the sine and cosine term. Also, the ripple frequency should have the same frequency of the grid. Moreover, the synchronous $d$-axis voltage does not include the ripple components related to the scaling error as shown in (8). In other words, the scaling error does not cause the distortion in the estimated grid angle and estimated grid frequency but affects the estimated grid voltage amplitude related to the synchronous q-axis voltage. It only relates to the feedforward term at the output of the current controller [14]. Therefore, this paper is considered to reduce the only dc offset error.

\subsection{Proposed dc offset error compensation algorithm}

The dc offset error of synchronous $d$-axis voltage causes the distorted grid angle and grid frequency in the PLL system.

The frequency component of the synchronous $d$-axis voltage is same as the grid frequency. The existing method for the dc offset error value can be estimated by integrator operation of the grid frequency one period. As the result, the dc offset error can be compensated by reducing the ripple components of the synchronous $d$-axis voltage in a PLL. However, the existing integral compensator has a drawback that is a slow dynamic response because monitoring one period of the grid voltage is required. The proposed algorithm has a prompt dynamic response because the dc offset error is continuously estimated by transforming the $d$-axis voltage to SRF method without monitoring one cycle time of the grid voltage.

Fig. 4 shows the block diagram of the proposed dc offset compensation algorithm. This algorithm is replaced low pass filters in the general SRF PLL method. dc offset error is displayed as sine waveform. This error is converted to the virtual axis and the synchronous coordination through all pass filter, so that the dc offset can be instantaneously

PLL Block Diagram

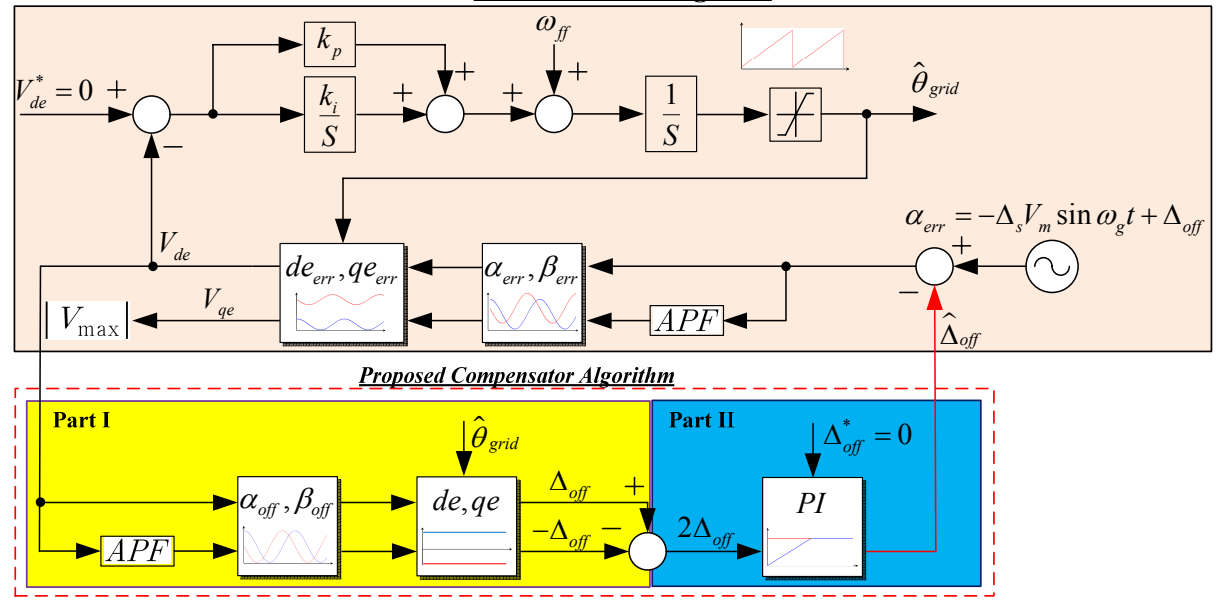

Fig. 4. Proposed dc offset error compensation algorithm block diagram 
investigated without monitoring the period of the grid frequency. Consequently, the dc offset error value $\Delta_{\text {off }}$ can be easily estimated by the synchronous $d q$-axis coordinate transformation of the synchronous $d$-axis voltage including the dc offset error value.

From (8), the synchronous $d$-axis voltage including dc offset error $V_{d e \text { eerr }}$ is composed of the sine and cosine term. Therefore, the dc offset error value is estimated by using the $V_{d e_{-} e r r}$. The $\alpha_{\text {off }}$ is set to (9).

$$
\alpha_{o f f}=V_{d e \_ \text {err }}
$$

where, $\alpha_{\text {off }}$ is sinusoidal wave caused by the dc offset error.

The two axes of the $\alpha_{\text {off }}, \beta_{\text {off }}$ are required for the synchronous coordinated conversion. Thus, the virtual voltage $\beta_{\text {off }}$ is delayed by $90^{\circ}$ from the $\alpha_{\text {off }}$ and it can be achieved by using the digital all pass filter. The rectangular coordinate system of the $\alpha_{\text {off }}, \beta_{\text {off }}$ can be calculated by (9).

$$
\left[\begin{array}{l}
\alpha_{o f f} \\
\beta_{o f f}
\end{array}\right]=\left[\begin{array}{l}
\Delta_{o f f} \cos \hat{\theta}_{g}+\Delta_{o f f} \sin \hat{\theta}_{g} \\
\Delta_{o f f} \sin \hat{\theta}_{g}-\Delta_{o f f} \cos \hat{\theta}_{g}
\end{array}\right]
$$

Considering (4), (10), the synchronous coordinated system can be written as (11).

$$
\begin{aligned}
& {\left[\begin{array}{l}
V_{d_{e_{-} o f f}} \\
V_{q e_{-} o f f}
\end{array}\right]=\left[\begin{array}{cc}
\cos \hat{\theta}_{g} & \sin \hat{\theta}_{g} \\
-\sin \hat{\theta}_{g} & \cos \hat{\theta}_{g}
\end{array}\right]\left[\begin{array}{l}
\alpha_{o f f} \\
\beta_{o f f}
\end{array}\right]} \\
& =\left[\begin{array}{cc}
\cos \hat{\theta}_{g} & \sin \hat{\theta}_{g} \\
-\sin \hat{\theta}_{g} & \cos \hat{\theta}_{g}
\end{array}\right]\left[\begin{array}{l}
\Delta_{\text {off }} \cos \hat{\theta}_{g}+\Delta_{o f f} \sin \hat{\theta}_{g} \\
\Delta_{o f f} \sin \hat{\theta}_{g}-\Delta_{\text {off }} \cos \hat{\theta}_{g}
\end{array}\right]
\end{aligned}
$$

$$
\begin{gathered}
=\left[\begin{array}{c}
\Delta_{\text {off }} \cos \hat{\theta}_{g} \cos \hat{\theta}_{g}+\Delta_{\text {off }} \sin \hat{\theta}_{g} \cos \hat{\theta}_{g} \\
-\Delta_{\text {off }} \cos \hat{\theta}_{g} \sin \hat{\theta}_{g}-\Delta_{\text {off }} \sin \hat{\theta}_{g} \sin \hat{\theta}_{g} \\
+\Delta_{\text {off }} \sin \hat{\theta}_{g} \sin \hat{\theta}_{g}-\Delta_{\text {off }} \cos \hat{\theta}_{g} \sin \hat{\theta}_{g} \\
+\Delta_{\text {off }} \sin \hat{\theta}_{g} \cos \hat{\theta}_{g}-\Delta_{\text {off }} \cos \hat{\theta}_{g} \cos \hat{\theta}_{g}
\end{array}\right] \\
=\left[\begin{array}{c}
\Delta_{\text {off }} \\
-\Delta_{\text {off }}
\end{array}\right] \quad \\
\hat{\Delta}_{\text {off }}=\Delta_{\text {off }}-\left(-\Delta_{\text {off }}\right)=2 \Delta_{\text {off }}
\end{gathered}
$$

The proposed compensator consists of two parts as shown in Fig. 4. The first part is the $d q$-axis coordinated transformation system used for estimating the dc offset error. $V_{d e_{e} e r r}$ is a component of sine waveform but $V_{d e_{-} e r r}$ and $V_{\text {qe off }}$ mean the positive and negative value of $\mathrm{dc}$ offset after the synchronous coordination conversion process. According to Eq. (12), these dc offset values calculate the difference each other and then double value of dc offset errors is obtained.

The other is the PI controller to decrease the dc offset error value. In addition, the output of the proposed PI controller is updated to get the constant of the exact dc offset error value in real time.

\section{Simulation}

Fig. 5 shows the simulation circuit for the SGC using the PSIM simulator. The rated power is designed as $3[\mathrm{~kW}]$. The parameter of SPC is displayed in Table 1.

Fig. 6 displays the simulation results considering the dc offset error of 5[V]. As shown in Fig. 6, the synchronous $d$-axis voltage is generated the same oscillation ripples with the grid frequency of $60[\mathrm{~Hz}]$.
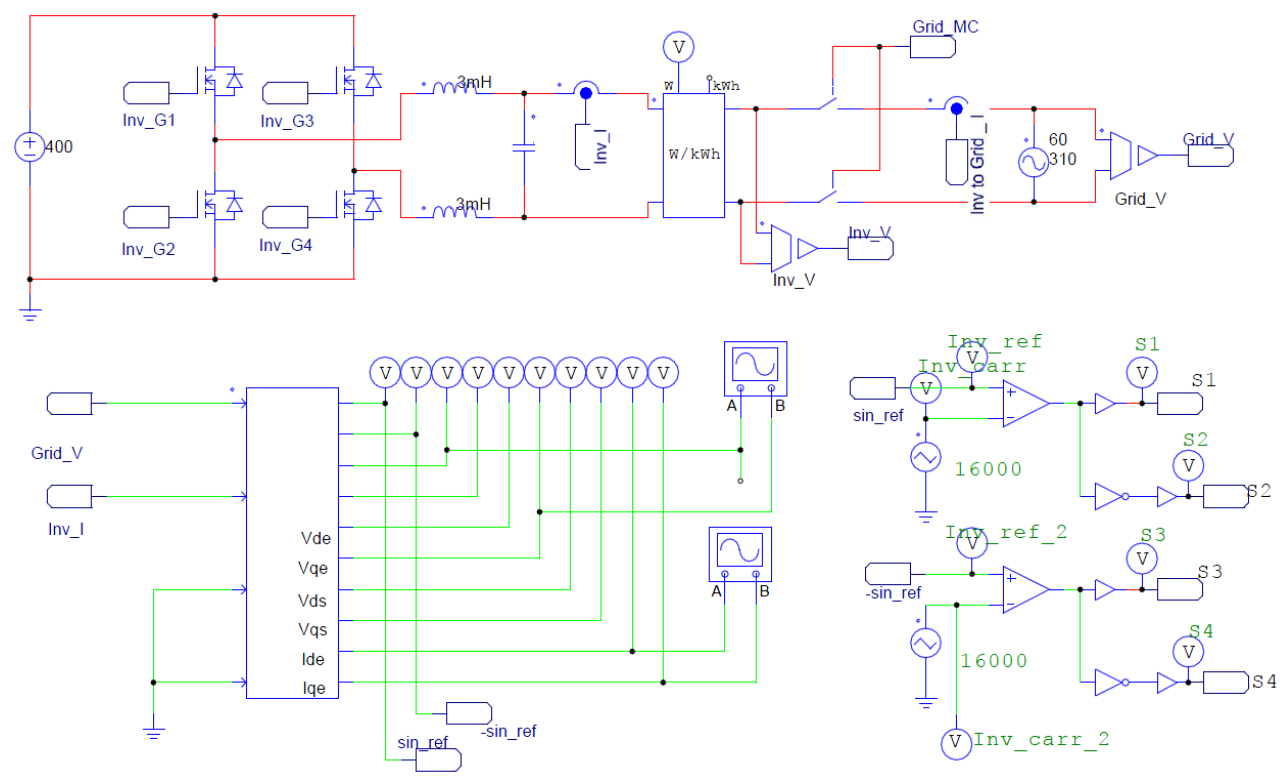

Fig. 5. Simulation circuit of the SGC by PSIM 
Table 1. The properties of the simulation parameter

\begin{tabular}{c|c}
\hline Properties & Details \\
\hline Rated power & $3[\mathrm{~kW}]$ \\
Switching frequency & $16[\mathrm{kHz}]$ \\
Grid voltage & $220[\mathrm{Vrms}]$ \\
Grid frequency & $60[\mathrm{~Hz}]$ \\
DC Link voltage & $370[\mathrm{~V}]$ \\
Simulation time step & $1[\mathrm{usec}]$ \\
\hline
\end{tabular}

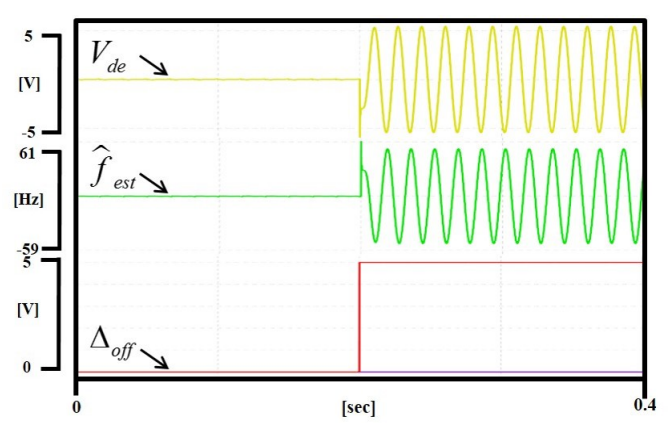

Fig. 6. Waveform after injecting the dc offset error $5[\mathrm{~V}]$ of the grid voltage

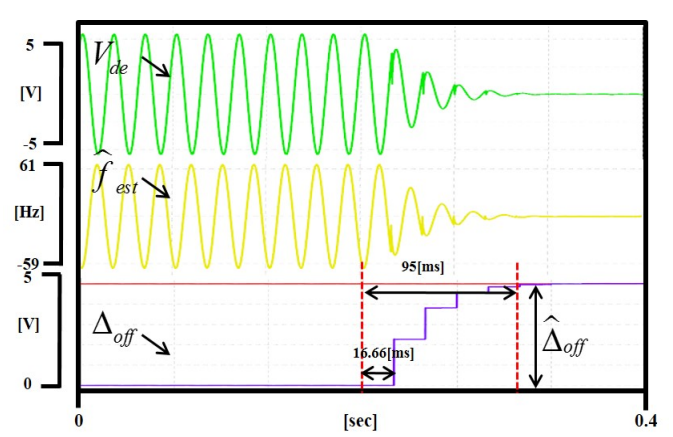

(a)

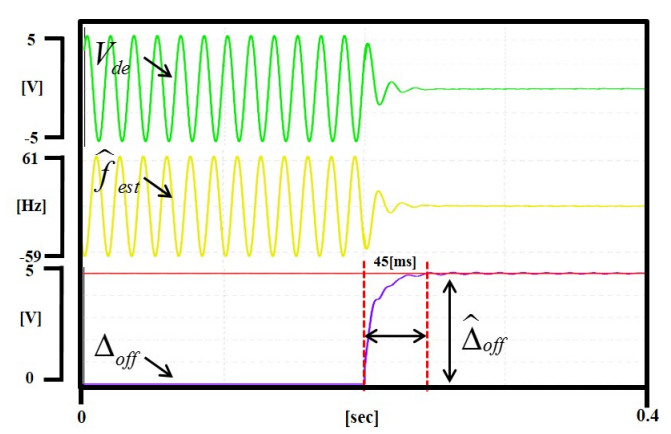

(b)

Fig. 7. Comparison of the simulation results with the conventional method: (a) Compensation waveform by the conventional method at $5[\mathrm{~V}]$ dc offset error of the grid voltage; (b) Compensation waveform by the proposed method at $5[\mathrm{~V}]$ dc offset error of the grid voltage

Fig. 7 - (a), (b) show the waveform comparing the dynamic characteristics of the proposed method and the existing method under $5[\mathrm{~V}]$ dc offset error.

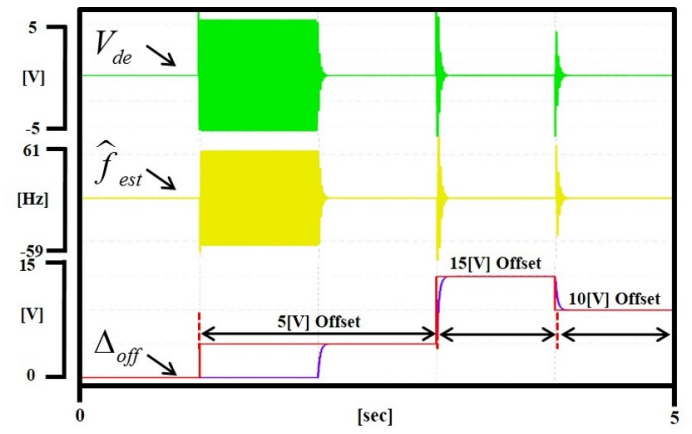

Fig. 8. Waveform according to changing the dc offset error

Fig. 7 (a) displays the result of the simulation using the general integration method. The compensation value is compensated in every one period of $60[\mathrm{~Hz}]$ because the process is that one period of the grid frequency is integrated and then the dc offset is detected. Therefore, the dc offset error is shown as the step curve. The time of the compensation is $95[\mathrm{~ms}]$.

Fig. 7 (b) illustrates the result of the dc offset inst antaneous compensation using the proposed coordination transformation. With the proposed method, the waveform of dc offset error is the continual curve because dc offset is instantly detected and compensated. The time of the compensation is $45[\mathrm{~ms}]$. Therefore, the proposed method can compensate the dc offset error faster than the existing method. It is proved that the dynamic characteristic of the proposed method is better than that of the general method.

Fig. 8 displays the dc offset error compensation performance of the proposed algorithm. When applied to 5 [V] dc offset error in the grid voltage at 1 second, the pulsation is seriously generated in maintained the constant synchronous $d$-axis voltage. The proposed algorithm is immediately started the dc offset error compensation in real time when 2 seconds. At this time, the pulsation of the synchronous $d$-axis voltage is reduced because of the compensation values and the dc offset error values the same. The dc offset error value is changed to $15[\mathrm{~V}]$ at 3 seconds. Finally, the dc offset error value is reduced to 10[V] when 4 seconds. Although the dc offset error value is changed, the pulsation of the synchronous $d$-axis voltage is quickly deleted because the dc offset error value is immediately estimated.

\section{Experimental Results}

The proposed dc offset error compensation method of synchronous $d$-axis voltage has been verified on a $3[\mathrm{~kW}]$ SGC. This converter is designed based on the 32bit DSP control system operating at $10[\mathrm{kHz}]$. The specification of the experiment is equated to that of the simulation. The prototype of a $3[\mathrm{~kW}] \mathrm{SGC}$ is shown in Fig. 9.

Fig. 10 shows the experimental results considering the variation of $5[\mathrm{~V}] \mathrm{dc}$ offset error. After the dc offset error is 


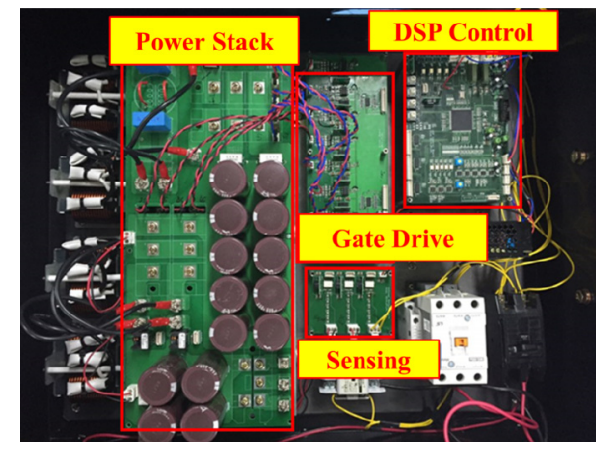

Fig. 9. Prototype of a $3[\mathrm{~kW}]$ single-phase grid-connected converter

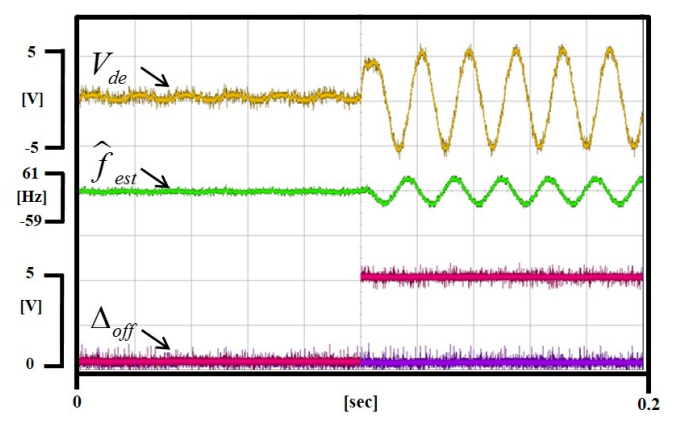

Fig. 10. Experimental results under $5[\mathrm{~V}]$ dc offset error of the grid voltage

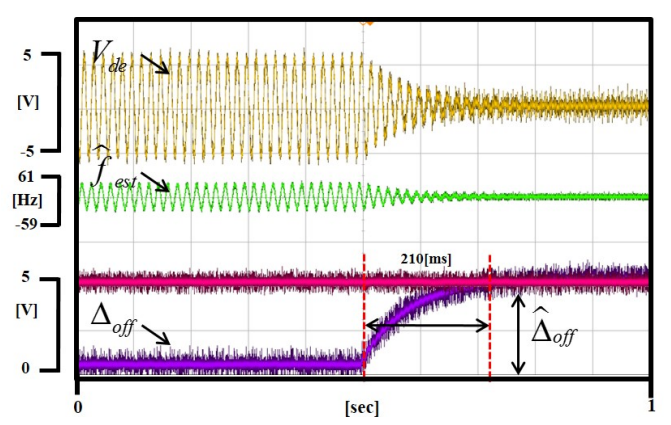

(a)

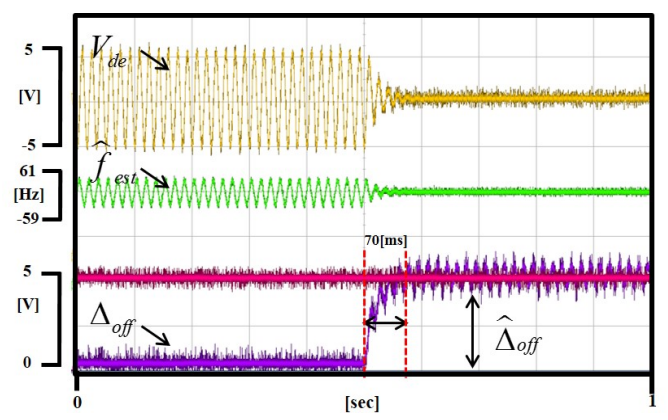

(b)

Fig. 11. Comparison of the experimental results with the conventional method (a) Compensation waveform by the conventional method at $5[\mathrm{~V}]$ dc offset error of the grid voltage (b) Compensation waveform by the proposed method at $5[\mathrm{~V}]$ dc offset error of the grid voltage

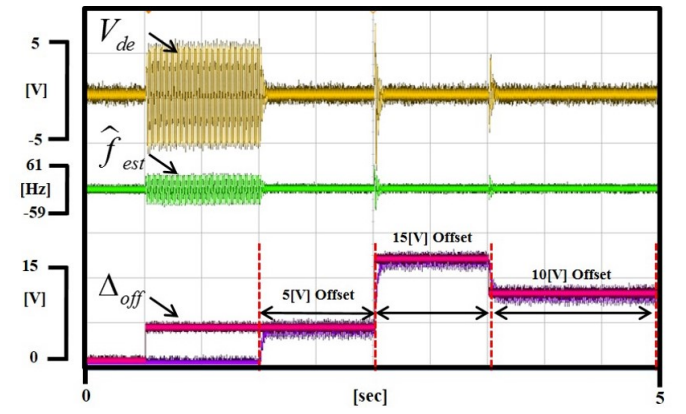

Fig. 12. Waveform according to changing the dc offset error

injected, the pulsation of the synchronous $d$-axis voltage is generated like simulation results of the Fig 6 .

The ripple component of the synchronous $d$-axis voltages is perfectly removed by the proposed compensation method as shown in Fig. 11. Fig. 11-(a) presents experimental results of the existing integral method, the dc offset error compensation time is 210 [ms]. Fig. 11-(b) illustrates experimental results of the proposed method, the dc offset error compensation time is $70[\mathrm{~ms}]$.

Fig. 12 displays the dc offset error compensation performance of the proposed algorithm. The experimental conditions are the same as the simulation.

\section{Conclusions}

A dc offset error compensation algorithm for the synchronous reference frame PLL of the single-phase gridconnected converter systems is proposed. The ripple is generated into the grid frequency and the voltage of the synchronous $\mathrm{d}-\mathrm{q}$ axis by the grid voltage detection process including $\mathrm{dc}$ offser. This ripple is clarified as the sine waveform contained the value of dc offset by the equations. When the ripple is occurred in the synchronous d-axis, the grid angle estimate should be contained the error due to generating the error in the PLL process.

The existing method has the disadvantage of the slow response characteristic. This is because the period of the grid frequency is integrated for detecting the value of $\mathrm{dc}$ offset, and then the detected value is compensated through PI controller.

In this paper, the dynamic characteristics of the proposed dc offset compensation method is much better than the existing method because this method can instantly investigate the value of dc offset through the coordination transformation and this value is compensated by using PI controller. The validity of the proposed algorithm is proved by the simulation and experimental test.

\section{Acknowledgements}

This work was supported by the Kyungnam University 
Foundation Grant, 2015.

\section{References}

[1] Yuan Wu, V. K. N. Lau, D. H. K. Tsang, Li Ping Qian and Limin Meng, "Optimal Energy Scheduling for Residential Smart Grid With Centralized Renewable Energy Source," IEEE. Systems Journal, Vol. 8, No. 2, pp. 562-576, Jun. 2014.

[2] S. R. Bull, "Renewable Energy today and tomorrow," IEEE. Proceedings, Vol. 89, No. 8, pp. 1216-1226, Aug. 2002.

[3] Y. M. Atwa, "Optimal Renewable Resources Mix for Distribution System Energy Loss Minimization," IEEE Trans. Power Systems, Vol. 25, No. 1, pp. 360370, Feb. 2010.

[4] B. Kroposki, P. K. Sen and K. Malmedal, "Optimum Sizing and Placement of Distributed and Renewable Energy Sources in Electric Power Distribution Systems," IEEE Trans. Industry Applications, Vol. 49, No. 6, pp. 2741-2752, Nov/Dec. 2013.

[5] M. Aiello, A. Cataliotti, V. Cosentino, and S. Nuccio, "Synchronization techniques for power quality instruments," IEEE Trans. Instrum. Meas., Vol. 56, No. 5, pp. 1511-1519, Oct. 2007.

[6] A. Cataliotti, V. Cosentino, and S.Nuccio, "A phaselocked loop for the synchronization of power quality instruments in the presence of stationary and transient disturbances," IEEE Trans. Instrum. Meas, Vol. 56, No. 6, pp. 2232-2239, Dec. 2007.

[7] G. Wang, H. Zhan, G. Zhang, X. Gui, and D. Xu, "Adaptive compensation method of position estimation harmonic error for EMF-based observer in sensorless IPMSM drives," IEEE Trans. Power Electron., Vol. 29, No. 6, pp. 3055-3064, Jun. 2014.

[8] R. Teodorescu, M. Liserre, and P. Rodriguez, "Grid Converters for Photovoltaic and Wind Power System," Chichester, U.K.: Wiley, 2011.

[9] C. Subranmanian, R. Kanagaraj, "Rapid Tracking of Grid Variables Using Prefiltered Synchonous Reference Frame PLL," IEEE Trans. Instrum. Meas, Vol. 64, No. 7, pp. 1826-1836, July. 2015.

[10] Behzad Mirzaeian Dehkordi, Amir Farrokh Payam, Mohammad Naser Hashemnia and Seung-Ki Sul, "Design of an Adaptive Backstepping Controller for Doubly-Fed Induction Machine Drives," IEEE Trans Power Electron, Vol. 9, No. 3, pp. 343-353, May. 2009.

[11] Sol-Bin Lee, Kyo-Beum Lee, Dong-Choon Lee and Jang-Mok Kim, "An Improved Control Method for a DFIG in a Wind Turbine under an Unbalanced Grid Voltage Condition," Journal of Electrical Engineering \& Technology, Vol. 5, No. 4, pp. 614-622, 2011.

[12] K. De Brabandere, T. Loix, K. Engelen, B. Bolsens, J. Van den Keybus, J. Driesen and R. Belmans,
"Design and Operation of a Phase-Locked Loop with Kalman Estimator-Based Filter for Single-Phase Applications," IEEE Industrial Electronics (IECON) 2006, pp. 525-530, 2006.

[13] Xiaorui Guo, Jifeng Guo, "A New Synchronization Algorithm for Grid-Connected Inverters," Applied Mathematics \& Information Sciences, Vol. 8, No. 2, pp. 869-875, Mar. 2014.

[14] U. A. Miranda, M. Aredes, and L. G. B. Rolim, "A dq synchronous reference frame control for single-phase converters," in Proc. PESC, 2005, pp. 1377-1381.

[15] S. H. Hwang, L. Liu and J. M. Kim, "DC offset error compensation for synchronous reference frame PLL in single-phase grid-connected converters," IEEE Trans. Power Electron, Vol. 27, No. 8, pp. 3467-3471, Aug. 2012.

[16] F. Blaabjerg, R. Teodorescu, M. Liserre and A. V. Timbus, "Overview of Control and Grid Synchronization for Distributed Power Generation Systems," IEEE Trans. Industrial Electronics, Vol. 53, No. 5, pp. 1398-1409, Oct. 2006.

[17] V. Kaura, V. Blasko, "Operation of a phase locked loop system under distorted utility conditions," IEEE Trans. Industry Applications, Vol. 33, No. 1, pp. 5863, Jan/Feb. 1997.

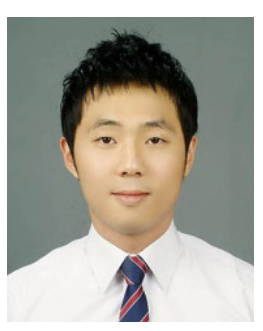

Chang-Seok Park was born in Changwon, Korea, in 1987. He received the B.S., M.S. degree in electrical engineering from Kyungnam University, Changwon, Korea, in 2012, 2014 respectively. $\mathrm{He}$ is currently a Ph.D. student at Kyungnam University. His main research interests are energy conversion machine and power conversion device design, control and application.

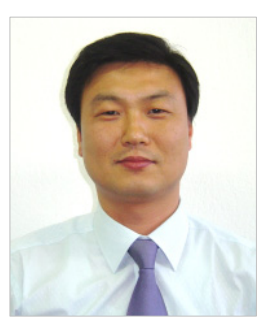

Tae-Uk Jung was born in Masan, Korea, in 1970. He received the B.S., M.S. and Ph.D. degrees in electrical engineering from Busan National University, Busan, Korea, in 1993, 1995 and 1999 , respectively. Between 1996 and 2005, he was a Chief Research Engineer with Laboratory of LG Electronics, Korea. Between 2006 and 2007, he was a Senior Research Engineer of Korea institute of Industrial Technology, Korea. Since 2007, he has been with Kyungnam University as a Professor. Prof. Jung is a member of the Institute of Electrical Engineers of Korea (KIEE) and IEEE. 